ASITP-94-26

August, 1994.

\title{
Standard Model
}

\section{With Higgs As Gauge Field On Fourth Homotopy Group}

\author{
Han-Ying Guo, Jian-Ming Li and Ke Wu \\ a. CCAST (World Laboratory), P.O. Box 8730, Beijing 100080, China; \\ c. Institute of Theoretical Physics, Academia Sinica, P.O. Box 2735, Beijing 100080, China.[2]
}

\begin{abstract}
Based upon a first principle, the generalized gauge principle, we construct a general model with $G_{L} \times G_{R}^{\prime} \times Z_{2}$ gauge symmetry, where $Z_{2}=\pi_{4}\left(G_{L}\right)$ is the fourth homotopy group of the gauge group $G_{L}$, by means of the non-commutative differential geometry and reformulate the Weinberg-Salam model and the standard model with the Higgs field being a gauge field on the fourth homotopy group of their gauge groups. We show that in this approach not only the Higgs field is automatically introduced on the equal footing with ordinary Yang-Mills gauge potentials and there are no extra constraints among the parameters at the tree level but also it most importantly is stable against quantum correlation.
\end{abstract}

\footnotetext{
${ }^{1}$ Work supported in part by The National Natural Science Foundation of China.

${ }^{2}$ Mailing address.
} 


\section{Introduction}

Unlike Yang-Mills gauge fields, Higgs fields and Yukawa couplings seem to be artificial although they play a very important role in modern QFT. Eventually, the price paid for them is the beauty of the gauge principle. How to regain the beauty of the gauge principle is one of the most intriguing problems in modern QFT.

Recently, we have generalized the ordinary Yang-Mills gauge theory in order to take both Lie groups and discrete groups as gauge groups [1,2] and completed an approach to this generalized gauge theory coupled to the fermions in the spirit of non-commutative geometry $[4,5]$. We have shown that Higgs fields are such gauge fields with respect to discrete gauge symmetry over 4-dimensional space-time $M^{4}$ and the Yukawa couplings between Higgs and fermions may automatically be introduced via generalized covariant derivatives. In this approach, Higgs appears as discrete fields on the equal footing with ordinary Yang-Mills fields over spacetime $M^{4}$. In other wards, the beauty of the gauge principle may be regained. Of course, how to understand the physical meaning of the discrete group to be gauged is a most crucial point in this approach. On the other hand, like other approaches [6-11] based upon the non-commutative differential geometry do not survive the standard quantum correlation [12], the approach in $[1,2]$ may also be unstable against the standard quantum correlation unless there is certain special mechanism to guarantee its stability.

In the letter [3], we have presented an $S U(2)$ generalized gauge field model with the Higgs mechanism and shown that it is able to get rid of all those problems based upon a first principle, the generalized gauge principle. The key point is that we have taken into account the fourth homotopy group of $S U(2)$ as a discrete gauge group on the footing with the Yang-Mills gauge group $S U(2)$. It is well known that the fourth homotopy group of $S U(2)$ is non-trivial, $\pi_{4}(S U(2))=Z_{2}$ [13], i.e. the gauge transformations of $S U(2)$ may be divided into two different equivalence classes. Once the Yang-Mills fields for the gauge group $S U(2)$ is introduced, the role played by its fourth homotopy group must be taken into account. In view of the generalized Yang-Mills gauge theory [1] based upon the non-commutative differential geometry, we should also introduce the generalized gauge field with respect to this internal discrete group $\pi_{4}(S U(2))$ due to the fact that the gauge transformations depend on its elements. Although there 
are several remarkable advantages in this model [3], but it is not phenomenologically realistic.

In this paper, we generalize the model presented in [3] to the realistic cases, such as the Weinberg-Salam model and the standard model. We show that the most responsible internal discrete symmetry for the Higgs, say, in the standard model is the forth homotopy group of the gauge groups, i.e. $\pi_{4}(S U(3) \times S U(2) \times U(1))=\pi_{4}(S U(2))=Z_{2}$. Similar to the model given in [3], there are several remarkable advantages in this approach. Firstly, it is a most natural choice of the discrete group for the Higgs and secondly it indicates that why the Higgs in the standard model is an $S U(2)$ doublet and $S U(3)$ singlet. Most importantly, it is stable against quantum correlation. We will discuss these issues at the end of this paper.

In what follows, we first construct a general model with $G_{L} \times G_{R}^{\prime} \times Z_{2}$ gauge symmetry, where $Z_{2}$ is the fourth homotopy group of the gauge group $G_{L} \times G_{R}^{\prime}$, i.e. $\pi_{4}\left(G_{L} \times G_{R}^{\prime}\right)$, because we pay our attention on the case of $\pi_{4}\left(G_{R}^{\prime}\right)=0$, then $\pi_{4}\left(G_{L} \times\right.$ $\left.G_{R}^{\prime}\right)=\pi_{4}\left(G_{L}\right)$. By means of the generalized gauge theory formulation [1] in the section 2. We also show that the Higgs mechanism is automatically included on the equal footing with ordinary Yang-Mills gauge fields and there are no extra constraints at the tree level among the coupling constants and mass parameters under suitable normalization. In the section 3, we reformulate the Weinberg-Salam model with Higgs being taken as the discrete gauge field on $\pi_{4}\left((S U(2) \times U(1))=Z_{2}\right.$. Then we deal with the standard model in the section 4. Finally, we end with some discussions and remarks. In the Appendix, we briefly introduce the non-commutative calculus on the discrete groups and show the Higgs fields is the gauge potentials with respect to the discrete gauge groups, while the Higgs potential may be given by a Lagrangian of the Yang-Mills type.

\section{$2 \quad$ A model with $G_{L} \times G_{R}^{\prime} \times Z_{2}$-gauge symmetry}

Let us first construct a model of the $G_{L} \times G_{R}^{\prime} \times Z_{2}$-gauge symmetry, where $\pi_{4}\left(G_{R}^{\prime}\right)=0$ and $Z_{2}$ is taken to be the fourth homotopy group of the gauge group $G_{L}$, i.e. $\pi_{4}\left(G_{L}\right)=$ $Z_{2}$, an intrinsic internal discrete group of the model. Namely, the gauge transformations of $G_{L}$ may be divided into two different equivalence classes. Consequently, All leptons 
$\psi(x, h), h \in Z_{2}$, Yang-Mills gauge potentials $A_{\mu}(x, h)$ of the gauge group $G_{L} \times G_{R}^{\prime}$ and Higgs $\Phi(x, h)$ with respect to the discrete gauge group $\pi_{4}\left(G_{L}\right)=Z_{2}$ included in this model of the generalized Yang-Mills type should be divided with respect to two elements of $\pi_{4}\left(G_{L}\right)=Z_{2}$. The construction of the model is based upon the generalized Yang-Mills gauge theory by means of the non-commutative differential geometry. It combines both the Yang-Mills gauge potentials and the Higgs as a kind of generalized Yang-Mills gauge potentials. For the details of this formalism, it is referred to [1] and some relevant notions are briefly introduced in the Appendix.

Let us regard those fields as elements of function space on $M^{4}$ as well as on $G_{L} \times G_{R}^{\prime} \times$ $Z_{2}$ and assign them into two sectors according to two elements of $\pi_{4}\left(G_{L}\right)=Z_{2}=\{e, r\}$. The $\pi_{4}\left(G_{L}\right)=Z_{2}$ symmetry requires that

$$
L_{\mu}(x, r)=U L_{\mu}(x, e) U^{-1}-\frac{i}{g} U \partial_{\mu} U^{-1},
$$

where $U(x)$ is a topologically nontrivial gauge transformation. Correspondingly, the left handed fermions should also be set down at these two elements noted as $L^{e}$ and $L^{r}$ respectively. Namely, there is a $Z_{2}$ symmetry between $L^{e}$ and $L^{r}$ :

$$
\psi(x, r)=\psi(x)^{U}=R_{r} \psi(x, e)=U(x) \psi, \quad \psi(x, e)=\psi(x)=R_{r} \psi(x, r)=U^{-1} \psi(x)^{U}
$$

As for the right handed fermions, we may take $R^{r}=R^{e}=R$. Therefor, we have

$$
\begin{gathered}
\psi(x, e)=\psi(x)=\left(\begin{array}{c}
L \\
R
\end{array}\right) ; \quad \psi(x, r)=\psi^{r}(x)=\left(\begin{array}{c}
L^{r} \\
R
\end{array}\right) \\
A_{\mu}(x, e)=A_{\mu}(x)=\left(\begin{array}{cc}
L_{\mu} & 0 \\
0 & R_{\mu}
\end{array}\right) ; \quad A_{\mu}(x, r)=A_{\mu}^{r}(x)=\left(\begin{array}{cc}
L_{\mu}^{r} & 0 \\
0 & R_{\mu}
\end{array}\right) \\
\Phi(x, e)=\Phi(x)=\left(\begin{array}{cc}
\frac{\mu}{\lambda} & -\phi \\
-\phi^{r \dagger} & \frac{\mu}{\lambda}
\end{array}\right) ; \Phi(x, r)=\Phi^{r}(x) .
\end{gathered}
$$

with the properties

$$
L^{r}=U L, \phi^{r}=U \phi, U U^{\dagger}=1
$$

$U$ is a non-trivial gauge transformation of $G_{L}$. In (2.1), $L(R)$ is the left (right) handed fermion, $L_{\mu}\left(R_{\mu}\right)$ the gauge potential valued on the Lie algebra of the gauge group $G_{L}\left(G_{R}^{\prime}\right), \mu$ and $\lambda$ two constants.

From the assignments (2.1), it is easy to see that the field contents of the model is of $Z_{2}$ symmetry and the Higgs in such a model may be regarded as the gauge field 
with respect to the gauged $Z_{2}$. However, it should be mentioned that the assignments (2.1) not only assign the fields to the elements of $Z_{2}$ but also imply that all fields are arranged into certain matrices. In fact, this aspect of the arrangements is nothing to do with discrete gauge symmetry but for convenience in the forthcoming calculation. Of course, it must be kept in mind that this is a working hypothesis and sometimes one should avoid certain extra constraints coming from this working hypothesis.

From the general framework in [1], it follows the generalized connection one-form

$$
A(x, h)=A_{\mu}(x, h) d x^{\mu}+\frac{\lambda}{\mu} \Phi(x, h) \chi, \quad h \in Z_{2},
$$

where $\chi$ denotes $\chi^{r}$ in the Appendix, and the generalized curvature two-form

$$
\begin{aligned}
F(h) & =d A(h)+A(h) \otimes A(h) \\
& =\frac{1}{2} F_{\mu \nu}(h) d x^{\mu} \wedge d x^{\nu}+\frac{\lambda}{\mu} F_{\mu r}(h) d x^{\mu} \otimes \chi+\frac{\lambda^{2}}{\mu^{2}} F_{r r}(h) \chi \otimes \chi .
\end{aligned}
$$

Using the above assignments, we get

$$
\begin{aligned}
F(x, e) & =F^{r}(x, r) \\
& =\frac{1}{2}\left(\begin{array}{cc}
L_{\mu \nu} & 0 \\
0 & R_{\mu \nu}
\end{array}\right) d x^{\mu} \wedge d x^{\nu}+\frac{\lambda}{\mu}\left(\begin{array}{cc}
0 & -D_{\mu} \phi \\
-\left(D_{\mu} \phi^{\dagger}\right)^{r} & 0
\end{array}\right) d x^{\mu} \otimes \chi \\
& +\frac{\lambda^{2}}{\mu^{2}}\left(\begin{array}{cc}
\phi \phi^{\dagger}-\frac{\mu^{2}}{\lambda^{2}} & 0 \\
0 & \phi^{r \dagger} \phi^{r}-\frac{\mu^{2}}{\lambda^{2}}
\end{array}\right) \chi \otimes \chi ;
\end{aligned}
$$

where

$$
D_{\mu} \phi=\partial_{\mu} \phi+L_{\mu} \phi-\phi R_{\mu}
$$

Having these building blocks, we may introduce the generalized gauge invariant Lagrangian with respect to each element of $\pi_{4}\left(G_{L}\right)=Z_{2}$, then take the Haar integral of them over $Z_{2}$ to get the entire Lagrangian of the model. Under certain consideration on the normalization in the Lagrangian, we may get a Lagrangian without any extra constraints among the coupling constants and the mass parameters at the tree level.

For the Lagrangian of the bosonic sector with respect to each element of $Z_{2}$, we have

$$
\begin{aligned}
\mathcal{L}_{Y M-H}(x, e)= & \mathcal{L}_{Y M-H}^{r}(x, r) \\
= & -\frac{1}{4 N_{L}} \operatorname{Tr}_{L}\left(L_{\mu \nu} L^{\mu \nu}\right)-\frac{1}{4 N_{R}} \operatorname{Tr}_{R}\left(R_{\mu \nu} R^{\mu \nu}\right) \\
& +\frac{2}{N} \eta \frac{\lambda^{2}}{\mu^{2}} \operatorname{Tr}\left(D_{\mu} \phi(x)\right)\left(D^{\mu} \phi(x)\right)^{\dagger} \\
& -\frac{2}{N} \eta^{2} \frac{\lambda^{4}}{\mu^{4}} \operatorname{Tr}\left(\phi(x) \phi(x)^{\dagger}-\frac{\mu^{2}}{\lambda^{2}}\right)^{2}+\text { const }
\end{aligned}
$$


where $N_{L}, N_{R}$ and $N$ are normalization constants introduced here to avoid some extra constraints from the matrix arrangement in (2.1), $\eta$ is a metric parameter defined by $\eta=<\chi, \chi>, \operatorname{Dim}(\eta)=\mu^{2}$. Here we suppose both $G_{L}$ and $G_{R}^{\prime}$ be semi-simple. Eventually, this is not necessary. For example, in the case of the Weinberg-Salam model and the standard model, $G_{L}$ is $S U(2)_{L} \times U(1)_{Y}$ and $S U(3)_{c} \times S U(2)_{L} \times U(1)_{Y}$ respectively. In those cases, we must change the way of taking normalization in order to avoid some extra constraints from the matrix arrangement (2.1).

For the fermionic sector, the Lagrangian with respect to each element of $Z_{2}$ may also be given as follows:

$$
\begin{aligned}
\mathcal{L}_{F}(x, e) & =\mathcal{L}_{F}^{r}(x, r) \\
& =i \bar{L} \gamma^{\mu}\left(\partial_{\mu}+L_{\mu}\right) L+i \bar{R} \gamma^{\mu}\left(\partial_{\mu}+R_{\mu}\right) R+\lambda\left(\bar{L} \phi R+\bar{R} \phi^{\dagger} L\right) .
\end{aligned}
$$

It is easy to get the entire Lagrangian for the model:

$$
\mathcal{L}(x)=\frac{1}{2} \sum_{h=e, r}\left\{\mathcal{L}_{F}(x, h)+\mathcal{L}_{Y M-H}(x, h)\right\}
$$

It is easy to see that first this is a Lagrangian with the Higgs mechanism of spontaneously symmetry breaking type included automatically which will be studied in detail in the forthcoming sections and secondly there do not exist any extra constraints among the coupling constants and mass parameters which is different from other approaches $[6-11]$.

\section{The Weinberg-Salam Model}

It is well known that the fourth homotopy group of the gauge group $G_{L}$ in the WeinbergSalam model is $\pi_{4}\left(S U(2)_{L} \times U(1)_{Y}\right)=\pi_{4}\left(S U(2)_{L}\right)=Z_{2}$. As was mentioned before, once the Yang-Mills fields for the gauge groups $S U(2)_{L} \times U(1)_{Y}$ are introduced, the role played by their fourth homotopy group must be taken into account. In view of the generalized Yang-Mills gauge theory [1] based upon the non-commutative differential geometry, we should also introduce the (generalized) gauge field with respect to this internal discrete group $Z_{2}$ as well.

Now let the elements of $\pi_{4}\left(S U(2)_{L} \times U(1)_{Y}\right)=Z_{2}$ be $\left\{U_{e}, U_{r}\right\}$ where $U_{e}$ represents the first topologically trivial equivalence class of the gauge transformations and $U_{r}$ the 
second class which is topologically non-trivial. We may first assign the $S U(2)_{L} \times U(1)_{Y}$ gauge fields into two sectors with respect to these two elements as what we have done in the last section and make use of the formulation in the last section. where $U(x, e)=V(x) \in U_{e}, U(x, r)=U(x) V(x) U^{-1}(x) \in U_{e}$. The bosonic part of the Lagrangian may also be given.

To be concrete and for the sake of simplicity, let us consider the Weinberg-Salam model with one family of leptons only and assign leptons, Yang-Mills gauge potentials and Higgs into two sectors according to two elements of the group $\pi_{4}\left(S U_{L}(2) \times U_{Y}(1)\right)=$ $Z_{2}$ as follows:

$$
\begin{gathered}
\psi(x, e)=\left(\begin{array}{c}
L \\
R
\end{array}\right) ; \quad \psi(x, r)=\left(\begin{array}{c}
L^{U} \\
R
\end{array}\right) ; \\
A_{\mu}(x, e)=\left(\begin{array}{cc}
L_{\mu} & 0 \\
0 & R_{\mu}
\end{array}\right) ; \quad A_{\mu}(x, r)=\left(\begin{array}{cc}
L_{\mu}^{U} & 0 \\
0 & R_{\mu}
\end{array}\right) ; \\
\Phi(x, e)=\Phi^{\dagger}(x, r)=\left(\begin{array}{cc}
\frac{\mu}{\lambda} & -\phi \\
-\phi^{U^{\dagger}} & \frac{\mu}{\lambda}
\end{array}\right) ;
\end{gathered}
$$

where $L$ and $\phi$ are $S U(2)$ doublets, $R$ an $S U(2)$ singlet and

$$
\begin{gathered}
L(x)=\left(\begin{array}{c}
\nu_{l} \\
l
\end{array}\right), \quad R(x)=l_{R} ; \quad \phi(x)=\left(\begin{array}{c}
\phi^{+} \\
\phi^{0}
\end{array}\right) ; \\
L_{\mu}=-i g \frac{\tau_{i}}{2} W_{\mu}^{i}+i \frac{g^{\prime}}{2} B_{\mu}, \quad L_{\mu}^{U}(x, r)=U L_{\mu}(x, e) U^{-1}-\frac{i}{g} U \partial_{\mu} U^{-1}, \quad R_{\mu}=i g^{\prime} B_{\mu} .
\end{gathered}
$$

Thus

$$
\begin{gathered}
L_{\mu \nu}=-i g \frac{\tau_{i}}{2} W_{\mu \nu}^{i}+i \frac{g^{\prime}}{2} B_{\mu \nu} ; \quad R_{\mu \nu}=i g^{\prime} B_{\mu \nu} \\
D_{\mu} \phi=\left(\partial_{\mu}-i g \frac{\tau_{i}}{2} W_{\mu}^{i}-i \frac{g^{\prime}}{2} B_{\mu}\right) \phi .
\end{gathered}
$$

From the general model we have set up in the last section, we may directly get the Lagrangian. For the Yang-Mills gauge bosons and the Higgs in the Weinberg-Salam model, we have

$$
\begin{aligned}
\mathcal{L}_{Y M-H}(x) & =-\frac{1}{4 N_{L}} \frac{g^{2}}{2} W_{\mu \nu}^{i} W^{i \mu \nu}-\frac{1}{4 N_{Y}} \frac{3 g^{2}}{2} B_{\mu \nu} B^{\mu \nu} \\
& +\frac{2}{N}\left(\eta \frac{\lambda^{2}}{\mu^{2}} \operatorname{Tr}\left(D_{\mu} \phi(x)\right)\left(D^{\mu} \phi(x)\right)^{\dagger}\right. \\
& \left.-\frac{2}{N} \eta^{2} \frac{\lambda^{4}}{\mu^{4}}\left(\operatorname{Tr} \phi(x) \phi(x)^{\dagger}-\frac{\mu^{2}}{\lambda^{2}}\right)^{2}\right),
\end{aligned}
$$

where $N_{L}, N_{Y}$ and $N$ are the normalization constants with respect to $S U(2)_{L}, U(1)_{Y}$ and the Higgs sector. The normalization of the coefficients of each term results

$$
N_{L}=\frac{g^{2}}{2}, \quad N_{Y}=\frac{3 g^{2}}{2}, \quad N=2 \frac{\lambda^{2}}{\mu^{2}} \eta
$$


It should be point out that the normalization have been taken here is different from the one in the last section since the gauge group $G_{L}=S U_{L}(2) \times U_{Y}(1)$ is not semi-simple. Similarly, we may get the Lagrangian for leptons $\mathcal{L}_{F}(x)$ as follows:

$$
\begin{aligned}
\mathcal{L}_{F}(x)= & -i \bar{L}(x) \gamma^{\mu}\left(\partial_{\mu}+i g \frac{\tau_{i}}{2} W_{\mu}^{i}-i \frac{g^{\prime}}{2} B_{\mu}\right) L \\
& -i \bar{R}(x) \gamma^{\mu}\left(\partial_{\mu}-i g^{\prime} B_{\mu}\right) R \\
& -\lambda\left(\bar{L}(x) \phi(x) R(x)+\bar{R}(x) \phi(x)^{\dagger} L(x)\right) .
\end{aligned}
$$

Thus, the entire Lagrangian for the Weinberg-Salam model reads

$$
\mathcal{L}(x)=\mathcal{L}_{F}(x)+\mathcal{L}_{Y M-H}(x) .
$$

It is easy to see that the Higgs potential takes its minimum value at $\operatorname{Tr}\left(\phi \phi^{\dagger}\right)=\left(\frac{\mu}{\lambda}\right)^{2}$ and the continuous gauge symmetry will spontaneously be broken down when the vacuum expectation value (VEV) is taken as

$$
<\phi>=\left(\begin{array}{c}
0 \\
\frac{\rho_{0}}{\sqrt{2}}
\end{array}\right),
$$

where $\rho_{0}=\sqrt{2} \frac{\mu}{\lambda}$. Now we take the VEV of $\phi$ and introduce a new field $\eta(x)$ as the Higgs field in the model

$$
\phi=\left(\begin{array}{c}
0 \\
\frac{\rho_{0}+\rho(x)}{\sqrt{2}}
\end{array}\right),
$$

as well as the photon and $Z$ boson via $W$ bosons and the Weinberg angle

$$
\begin{gathered}
A_{\mu}=B_{\mu} \cos \theta_{W}+W_{\mu}^{3} \sin \theta_{W} \\
Z_{\mu}=B_{\mu} \sin \theta_{W}-W_{\mu}^{3} \cos \theta_{W} \\
g \sin \theta_{W}=g^{\prime} \cos \theta_{W}=\frac{g g^{\prime}}{\sqrt{g^{2}+g^{\prime 2}}}=e,
\end{gathered}
$$

where $e$ is the charge of the positron. Using these definitions, we get

$$
\sin ^{2} \theta_{W}=\frac{g^{\prime 2}}{g^{2}+g^{\prime 2}} \quad\left\{=\frac{N_{Y}}{3 N_{L}+N_{Y}}\right\}
$$

And we have

$$
\begin{aligned}
& \operatorname{Tr}\left\{D_{\mu} \phi\left(D_{\mu} \phi\right)^{\dagger}-\eta \frac{\lambda^{2}}{\mu^{2}}\left(\phi \phi^{\dagger}-\frac{\mu^{2}}{\lambda^{2}}\right)^{2}\right\} \\
& =\frac{1}{2} \partial_{\mu} \rho \partial^{\mu} \rho+\frac{g^{2}}{4}\left(\rho_{0}+\rho\right)^{2} W_{\mu}^{-} W_{\mu}^{+}+\frac{1}{8}\left(g^{2}+g^{\prime 2}\right)\left(\rho_{0}+\rho\right)^{2} Z_{\mu} Z_{\mu} \\
& \quad-\eta \frac{\lambda^{2}}{\mu^{2}} \rho^{2}\left(\rho_{0}^{2}+\rho_{0} \rho+\frac{\rho^{2}}{4}\right)+\text { const. }
\end{aligned}
$$

It is easy to see that only $A_{\mu}$ and $\nu_{l}$ remain massless while fermion $l$ together with $W^{ \pm}$ and $\mathrm{Z}$ become massive and following mass relations hold at the tree level:

$$
\begin{aligned}
& M_{\text {fermion }}=\mu, \quad M_{W}=\frac{1}{2} g \rho_{0}, \\
& M_{Z}=\frac{1}{2} \sqrt{g^{2}+g^{\prime 2}} \rho_{0}=M_{W} / \cos \theta_{w} \\
& M_{\text {Higgs }}=2 \sqrt{\eta} .
\end{aligned}
$$


It is easy to see that all these relations at the tree level are the same as the ones for the Weinberg-Salam model except the last one for the Higgs mass but different from what is given in [6]. The reason is that we have introduced two independent normalization constants $N_{L}, N_{Y}$ and $N$ in order to avoid some extra constraints from the matrix arrangement (1). In fact, if we would take $N_{L}=N_{Y}$ we could get the same constraint for the Weinberg angle in [6]. In other wards, as was mentioned in [1] the constraints in [6] are not essential but completely dependent on the working hypothesis. As for the Higgs mass given here at the tree level, it depends on the metric parameter $\eta$. If we let it free of choice, there is no constraint for the Higgs mass at all.

\section{Standard Model for Electroweak-Strong Inter- action}

We now turn to the standard model for the electroweak-strong interactions. This should be more realistic from both conceptual and phenomenological points of view. We take into account the colour degree of freedom together with the weak isospin and the weak hypercharge degrees of freedom for both leptons and quarks in three families. Similar to what we have done in the last section, we first introduce the gauge fields of the Yang-Mills gauge groups $S U(2)_{L} \times U(1)_{Y} \times S U(3)_{c}$ and assign them into two elements

of $\pi_{4}\left(S U(2)_{L} \times U(1)_{Y} \times S U(3)_{c}\right)=Z_{2}$ symmetry respectively. Such assignments for the fermions and the Higgs will be given according to their couplings to the Yang-Mills gauge fields as well.

The assignment for the fermions with respect to $\pi_{4}\left(S U(2)_{L} \times U(1)_{Y} \times S U(3)_{c}\right)=$ $Z_{2}=\{e, r\}$ may be taken as follows:

$$
\psi(x, e)=\left(\begin{array}{c}
L \\
R
\end{array}\right), \quad \psi(x, r)=\left(\begin{array}{c}
L^{\mathcal{U}} \\
R
\end{array}\right),
$$

with $L^{\mathcal{U}}=\mathcal{U} L=\left(\begin{array}{cc}U \otimes I_{3} \otimes I_{3}^{c} & \\ & U \times I_{3}\end{array}\right) L, \mathrm{U}$ the topologically non-trivial gauge 
transformation of $S U(2)_{L}$ and

$$
L=\left(\begin{array}{c}
u^{c} \\
\vdots \\
b^{c} \\
\nu_{e} \\
\vdots \\
\tau
\end{array}\right)_{L}^{G F C}, \quad R=\left(\begin{array}{c}
u^{c} \\
\vdots \\
b^{c} \\
e \\
\mu \\
\tau
\end{array}\right)_{R}
$$

Here superscript $c$ stands for the colour degree of freedom. Taking into account all strong and electroweak interactions among leptons and quarks according to (4.2), we assign the gauge fields as follows:

$$
A_{\mu}(x, e)=\left(\begin{array}{cc}
L_{\mu} & 0 \\
0 & R_{\mu}
\end{array}\right), \quad A_{\mu}(x, r)=\left(\begin{array}{cc}
L_{\mu}^{\mathcal{U}} & 0 \\
0 & R_{\mu}
\end{array}\right),
$$

where

$$
\begin{aligned}
& L_{\mu}=-\left(\begin{array}{ll}
\frac{i g}{2} \tau_{i}^{I} W_{\mu}^{i} \otimes I_{3}^{G} \otimes I_{3}^{C} & \\
& \frac{i g}{2} \tau_{i}^{I} W_{\mu}^{i} \otimes I_{3}^{G}
\end{array}\right) \\
& -i g B_{\mu}\left(\begin{array}{cc}
\frac{1}{6} I_{2} \otimes I_{3}^{G} \otimes I_{3}^{C} & \\
& -\frac{1}{2} I_{2} \otimes I_{3}^{G}
\end{array}\right)-\left(\begin{array}{cc}
I_{2} \otimes I_{3}^{G} \otimes \frac{i g_{2}}{2} G_{\mu}^{i} \lambda_{i}^{C} & \\
& 0
\end{array}\right), \\
& L_{\mu}^{\mathcal{U}}=\mathcal{U} L_{\mu} \mathcal{U}^{-1}+\mathcal{U} \partial_{\mu} \mathcal{U}^{-1}
\end{aligned}
$$

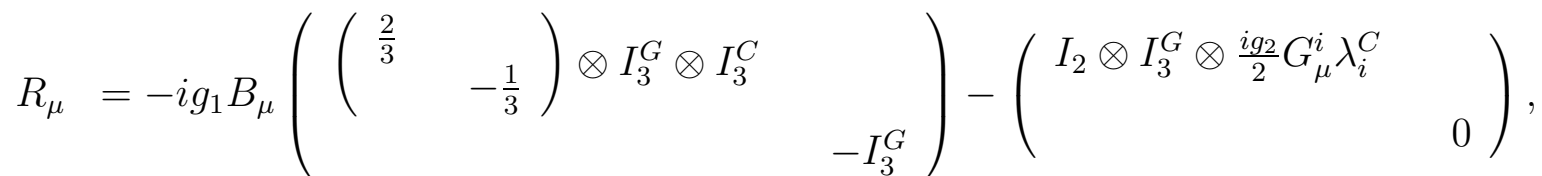

where $G_{\mu}^{i}, i=1, \cdots, 8$, are gluons, $\lambda_{i} 3 \times 3$ Gell-Mann matrices, and $I_{n} n \times n$ unit matrices. For the Higgs field, we take it as before

$$
\Phi(x, e)=\Phi^{\dagger}(x, r)=\left(\begin{array}{cc}
0 & \Phi(x) \\
(\mathcal{U} \Phi(x))^{\dagger} & 0
\end{array}\right) .
$$

But, $\Phi(x)$ field being gauge field with respect to $Z_{2}$-symmetry is more complicated:

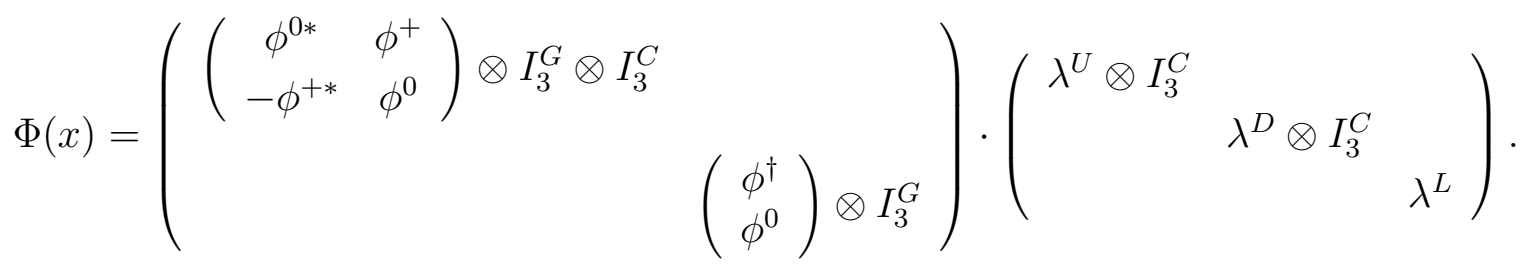

Here the blocks in the last matrix are $3 \times 3$ matrices in the space of generation, $\lambda^{U}, \lambda^{D}$ the matrices for quarks and $\lambda^{L}$ the matrices for leptons. These matrices play the role of the Yukawa coupling constants. 
Now we may write down the generalized connection one-form including both ordinary Yang-Mills potentials and the Higgs field on the equal footing and the generalized curvature two-form. Especially, the components $F_{\mu r}$ of the generalized field strength are the ordinary covariant derivative of the Higgs field as before:

$$
D_{\mu} \Phi=\partial_{\mu} \Phi+L_{\mu} \Phi-\Phi R_{\mu}
$$

Making use of the model in the section 2, we may get the Lagrangian as long as the normalization is suitably taken. The bosonic part of the entire gauge invariant Lagrangian, by some straightforward calculation, is

$$
\begin{aligned}
\mathcal{L}_{Y M-H}= & -\frac{1}{N}<F, \bar{F}> \\
= & -\frac{1}{4 N_{L}} 6 g^{2} W^{i \mu \nu} W^{i \mu \nu}-\frac{1}{4 N_{Y}} 10 g_{1}^{2} B_{\mu \nu} B^{\mu \nu}-\frac{1}{4 N_{c}} 6 g_{2}^{2} G_{\mu \nu}^{i} G^{i \mu \nu} \\
& +\frac{1}{N}\left\{2 \eta \frac{\sigma_{1}^{\prime}}{\mu^{2}}\left(D_{\mu} \pi\right)^{\dagger} D^{\mu} \pi-2 \eta^{2} \frac{\sigma_{2}^{\prime}}{\mu^{4}}\left(\pi^{\dagger} \pi-\frac{\sigma_{1}}{\sigma_{2}} \mu^{2}\right)^{2}\right\},
\end{aligned}
$$

where $N_{L}, N_{Y}, N_{c}$ and $N$ are normalization constants with respect to gauge fields W, $\mathrm{B}, \mathrm{G}$ and the Higgs sector respectively, the field $\pi$ is introduced as $\pi=\left(\begin{array}{c}\phi^{\dagger} \\ \phi^{0}\end{array}\right)$, and

$$
\begin{aligned}
& \sigma_{1}=\operatorname{Tr}\left(\begin{array}{ccc}
\lambda^{U} \lambda^{U^{\dagger}} \otimes I_{3}^{C} & \\
& \lambda^{D} \lambda^{D^{\dagger}} \otimes I_{3}^{C} & \\
& & \lambda^{L} \lambda^{L^{\dagger}}
\end{array}\right)^{2}, \\
& \sigma_{2}=\operatorname{Tr}\left(\begin{array}{ccc}
\lambda^{U} \lambda^{U^{\dagger}} \otimes I_{3}^{C} & \\
& \lambda^{D} \lambda^{D^{\dagger}} \otimes I_{3}^{C} & \\
& & \lambda^{L} \lambda^{L^{\dagger}}
\end{array}\right)^{2} .
\end{aligned}
$$

The normalization of the coefficients of the terms in the entire Lagrangian leads to that

$$
N_{L}=6 g^{2}, \quad N_{Y}=10 g_{1}^{2}, \quad N_{c}=6 g_{2}^{2}, \quad N=2 \frac{\sigma_{1}}{\mu^{2}} \eta .
$$

This gives rise to the following form for the Yang-Mills-Higgs Lagrangian

$$
\begin{aligned}
\mathcal{L}_{Y M-H}= & -\frac{1}{4} W_{\mu \nu}^{i} W^{i \mu \nu}-\frac{1}{4} B_{\mu \nu} B^{\mu \nu}-\frac{1}{4} G_{\mu \nu}^{i} G^{i \mu \nu} \\
& +\left(D_{\mu} \pi\right)^{\dagger} D^{\mu} \pi-\frac{\eta}{\mu^{2}} \frac{\sigma_{2}}{\sigma_{1}}\left(\pi^{\dagger} \pi-\frac{\sigma_{1}}{\sigma_{2}} \mu^{2}\right)^{2} .
\end{aligned}
$$

It is obviously that together with the Lagrangian of the usual gauge fields the kinetic energy of Higgs field and the interaction between Higgs field and the usual gauge fields are all included here.

It is easy to see that when $\pi$ field takes value

$$
|\pi|=\sqrt{\frac{\sigma_{1}}{\sigma_{2}}} \mu
$$


the Higgs potential is at its minimum. If we set the vacuum expectation value as

$$
<\pi>=\left(\begin{array}{c}
0 \\
\frac{\rho_{0}}{\sqrt{2}}
\end{array}\right), \quad v=\sqrt{\frac{2 \sigma_{1}}{\sigma_{2}}} \mu,
$$

the continuous gauge symmetry is broken down. Introducing a field $\rho(x)$

$$
\pi=\left(\begin{array}{c}
0 \\
\frac{\rho_{0}+\rho(x)}{\sqrt{2}}
\end{array}\right)
$$

and the photon $\mathrm{A}$ as well as the boson $\mathrm{Z}$

$$
\begin{aligned}
& A_{\mu}=\sin \theta_{w} W_{\mu}^{3}+\cos \theta_{w} B_{\mu}, \\
& Z_{\mu}=\cos \theta_{w} W_{\mu}^{3}-\sin \theta_{w} B_{\mu},
\end{aligned}
$$

where

$$
g \sin \theta_{w}=g^{\prime} \cos \theta_{w}=\frac{g g^{\prime}}{\sqrt{g^{2}+g^{\prime 2}}}=e,
$$

we may get spontaneous symmetry breaking version of (4.8).

For the fermions, we can also write down the Lagrangian in a way similar to what we have done for the model of leptons in the last subsection:

$$
\begin{aligned}
\mathcal{L}_{F}(x)= & \sum_{i} \bar{q}_{i} i \gamma^{\mu} D_{\mu} q_{i}+\sum_{i} \bar{l}_{i} i \gamma^{\mu} D_{\mu} l_{i} \\
& -\left\{\left(\bar{e}_{R} \bar{\mu}_{R} \bar{\tau}_{R}\right) \lambda^{L}\left(\begin{array}{c}
e_{L} \\
\mu_{L} \\
\tau_{L}
\end{array}\right)+\left(\bar{u}_{R}^{c} \bar{c}_{R}^{c} \bar{t}_{R}^{c}\right) \lambda^{U} \otimes I_{3}\left(\begin{array}{c}
u_{L}^{c} \\
c_{L}^{c} \\
t_{L}^{c}
\end{array}\right)\right. \\
& \left.+\left(\bar{d}_{R}^{c} \bar{s}_{R}^{c} \bar{b}_{R}^{c}\right) \lambda^{D} \otimes I_{3}\left(\begin{array}{c}
d_{L}^{c} \\
s_{L}^{c} \\
b_{L}^{c}
\end{array}\right)+h . c .\right\} \frac{\rho_{0}}{\sqrt{2}}\left(1+\frac{\rho}{\rho_{0}}\right)
\end{aligned}
$$

As is well known, both $\lambda^{L}$ and $\lambda^{U}$ may be diagnolized as

$$
\lambda^{L}=\left(\begin{array}{ccc}
\lambda_{e} & & \\
& \lambda_{\mu} & \\
& & \lambda_{\tau}
\end{array}\right), \quad \lambda^{U}=\left(\begin{array}{ccc}
\lambda_{u} & & \\
& \lambda_{c} & \\
& & \lambda_{t}
\end{array}\right)
$$

while $\lambda^{D}$ may be written as

$$
\lambda^{D}=V\left(\begin{array}{ccc}
\lambda_{d} & & \\
& \lambda_{s} & \\
& & \lambda_{b}
\end{array}\right) V^{\dagger}
$$

where $V$ is the Kobayashi-Maskawa matrix.

Since the mass of top quark is much heavier than other fermions, i.e. $m_{t} \gg m_{i}$, where $m_{i}$ is the mass for the fermion $i$ except $t$, we have

$$
\frac{\sigma_{2}}{\sigma_{1}^{2}}=\frac{1}{3}, \quad \frac{\sigma_{1}}{\sigma_{2}}=\frac{1}{\lambda_{t}^{2}}, \quad \epsilon=\sqrt{\frac{\eta}{\mu^{2}}}
$$


where $\lambda_{t}$ is the coupling constant corresponding to the top quark. Then the Lagrangian for the generalized gauge fields can be rewritten as

$$
\begin{aligned}
\mathcal{L}_{Y M-H}= & -\frac{1}{4} W_{\mu \nu}^{i} W^{i \mu \nu}-\frac{1}{4} B_{\mu \nu} B^{\mu \nu}-\frac{1}{4} G_{\mu \nu}^{i} G^{i \mu \nu} \\
& +\left(D_{\mu} \pi\right)^{\dagger} D^{\mu} \pi-\eta \frac{\lambda_{t}^{2}}{\mu^{2}}\left(\pi^{\dagger} \pi-\frac{\mu^{2}}{\lambda_{t}^{2}}\right)^{2}
\end{aligned}
$$

Consequently, when $\pi$ field takes value $|\pi|=\frac{\mu}{\lambda_{t}}$, the Higgs potential is at its minimum. If we set

$$
<\pi>=\left(\begin{array}{c}
0 \\
\frac{\rho_{0}}{\sqrt{2}}
\end{array}\right), \quad \rho_{0}=\frac{\sqrt{2}}{\lambda_{t}} \mu
$$

the symmetry $S U(2)_{L} \times U(1)_{Y}$ will spontaneously be broken down. Introducing new field $\rho$ to replace the field $\pi$ in eq.4.17), just as we have done in the last section, and adding the fermionic part through generalized covariant derivative, we get the final expression of the entire Lagrangian as follows

$$
\begin{aligned}
& \mathcal{L}(x)=\mathcal{L}_{F}(x)+\mathcal{L}_{Y M-H}(x) \\
& =\sum_{i} \bar{q}_{i} i \gamma^{\mu} D_{\mu} q_{i}+\sum_{i} \bar{l}_{i} i \gamma^{\mu} D_{\mu} l_{i}
\end{aligned}
$$

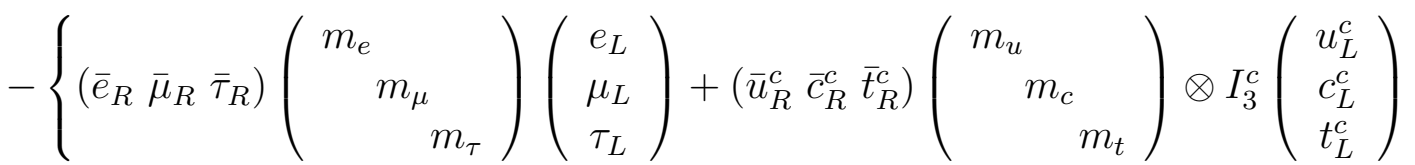

$$
\begin{aligned}
& \left.+\left(\begin{array}{lll}
\bar{d}_{R}^{c} & \bar{s}_{R}^{c} & \bar{b}_{R}^{c}
\end{array}\right) V\left(\begin{array}{cc}
m_{d} & \\
& m_{s} \\
& m_{b}
\end{array}\right) V^{\dagger} \otimes I_{3}^{c}\left(\begin{array}{c}
d_{L}^{c} \\
s_{L}^{c} \\
b_{L}^{c}
\end{array}\right)+\text { h.c. }\right\}\left(1+\frac{\rho}{\rho_{0}}\right) \\
& -\frac{1}{2} W_{\mu \nu}^{\dagger} W^{\mu \nu}-\frac{1}{4} Z_{\mu \nu} Z^{\mu \nu}-\frac{1}{4} A_{\mu \nu} A^{\mu \nu} \\
& +\frac{1}{2} \partial_{\mu} \rho \partial^{\mu} \rho+\frac{g^{2}}{4}\left(\rho_{0}+\rho\right)^{2} W^{-} W^{+}+\frac{g^{2}}{8 \cos \theta_{w}{ }^{2}}\left(\rho_{0}+\rho\right)^{2} Z_{\mu} Z^{\mu} \\
& -\frac{\eta}{\mu^{2}} \frac{\sigma_{2}}{\sigma_{1}}\left(\rho_{0}^{2} \rho^{2}+\rho_{0} \rho^{3}+\frac{\rho^{4}}{4}\right)
\end{aligned}
$$

It is easy to see that neutrinos, photon and gluons remain massless while other particles become massive. And we can also get the following mass relations,

$$
\begin{aligned}
& m_{W}=\frac{1}{2} g \rho_{0} \\
& m_{Z}=\frac{m_{W}}{\cos \theta_{w}} \\
& m_{\text {Higgs }} \approx 2 \sqrt{\eta} \\
& m_{t} \approx \mu
\end{aligned}
$$

Similar to the last section, it is easy to see that all these relations at the tree level are the same as the ones for the standard model except that for the Higgs mass. The 
Higgs mass given here also depends on the metric parameter $\eta$. If we let it free of choice, there is no constraint for it at all. Otherwise, if we would take

$$
\eta=\mu^{2}
$$

we could get

$$
M_{H}=2 m_{t} .
$$

However, there is no profound reason to do so.

\section{$5 \quad$ Concluding Remarks}

Now we summarize what we have done as follows:

We have first constructed a general model with $G_{L} \times G_{R}^{\prime} \times Z_{2}$ gauge symmetry, where $Z_{2}$ is $\pi_{4}\left(G_{L}\right)$, by means of the generalized gauge theory on both Lie groups and discrete groups. We have shown that the Higgs mechanism is automatically included in the generalized gauge theory and there are no constraints among the parameters at the tree level in this model. Then we have reformulated the Weinberg-Salam model and the standard model with the Higgs field being a gauge field with respect to the fourth homotopy group of the gauge groups, i.e. $\pi_{4}(S U(3) \times S U(2) \times U(1))=\pi_{4}(S U(2))=Z_{2}$.

It is worthy to point out that there are several advantages in this approach as was mentioned at the introduction of this paper. First of all, this $\pi_{4}(S U(2))=Z_{2}$ symmetry is a most natural internal symmetry to be gauged in these models in the sense of non-commutative differential calculus on the function space on $M^{4}$ as well as on $G_{L} \times G_{R}^{\prime} \times \pi_{4}\left(G_{L} \times G_{R}^{\prime}\right)=Z_{2}$. In fact, for these models, the fourth homotopy group of the gauge groups is already there and it should play certain role in the gauge theory. What we have done here is just to combine the ordinary Yang-Mills gauge theory with the non-commutative differential calculus in the function space on this discrete group to formulate a generalized gauge theory with Higgs and spontaneously symmetry breaking. In other wards, the Higgs mechanism should be introduced automatically at same footing with the ordinary Yang-Mills gauge field theory, if the role played by the fourth homotopy group of the gauge groups would be taken into account at very beginning. 
Secondly, it is also interesting to see that the mystery of the Higgs pattern in the standard model may be understood better. In fact, that $\pi_{4}(S U(3))=0, \pi_{4}(U(1))=$ 0 and $\pi_{4}(S U(2))=Z_{2}$ indicates that Higgs should play certain role for the $S U(2)$ gauge field and nothing to do for the $S U(3)$ gauge symmetry. Taking into account the properties of the fermions the Higgs in the standard model should be an $S U(2)$ doublet and $S U(3)$ singlet.

Finally, it is remarkable that the approach presented here with the fourth homotopy group of the gauge groups being the discrete gauge group is stable against quantum correlation. This is due to the following reasons. Firstly, there are no constraints among the parameters at the tree level so that we do not need to pay attention to them in the course of quantization and renormalization. Secondly, since the Higgs potential is automatically introduced in the generalized gauge theory, the $S U(2)$ gauge symmetry should be spontaneously broken down. Therefore, this $Z_{2}$ symmetry, the fourth homotopy group of the gauge symmetry in those models is also broken down as well. Consequently, what we got is, say, the same version as the ordinary standard model and we of course do not need to concern about this $Z_{2}$-gauge symmetry when we consider the quantum correlation of the model. Needless to say, this is a very important point different from other approaches to the Higgs by means of the non-commutative differential geometry. In fact, Connes like approaches [6-10] do not survive the quantum correlation [11].

In conclusion, the Higgs mechanism may be a part of a generalized Yang-Mills gauge theory as long as a global aspect, the fourth homotopy group, of the gauge group is taken into account in the sense of the non-commutative differential geometry. For the standard model the most natural and meaningful discrete symmetry on which the Higgs is a generalized gauge fields is just the fourth homotopy group of the gauge groups.

It is clear that the model presented in the section 2 may be generalized to the case of $\pi_{4}\left(G_{L} \times G_{R}^{\prime}\right)=Z_{2} \times Z_{2}$ and it may be applied to the left-right symmetric model. On the other hand, since the fourth homotopy group of $S U(5) /(S U(3) \times S U(2) \times U(1))$ is also non-trivial, it may play certain role in the $\mathrm{SU}(5)$-GUT together with the fourth homotopy group of $S U(3) \times S U(2) \times U(1)$. And all models of these kind may have the 
same advantages as the approach presented in this paper. Especially, all of them may be stable against quantum correlation. As for other discrete symmetries such as $C P T$ and so on, they may play other roles such as CP violation and so on. We will study these issues elsewhere.

\section{Appendix}

\section{Differential Calculus on Discrete Group G}

In this appendix, we briefly introduce some notions in the non-commutative differential calculus on the function space on discrete groups and show the Higgs may be regarded as the (generalized) gauge potential on the gauged discrete group. For the details, it is referred to Sitarz in [5] and our papers [1].

Let $\mathrm{G}$ be discrete group of size $N_{G}$, its elements are $\left\{e, g_{1}, g_{2}, \cdots, g_{N_{G}-1}\right\}$, and $\mathcal{A}$ the algebra of the all complex valued functions on G. In order to construct the first order differential calculus $\left(\Omega^{1}, d\right)$, one can give first the definition of its dual space $\mathcal{F}$, the vector space on $\mathcal{A}$ with basis $\partial_{i},\left(i=1, \cdots, N_{G}-1\right)$ as follows:

$$
\partial_{g}=f-R_{g} f, \quad g \in G^{\prime}, f \in \mathcal{A},
$$

where

$$
\left(R_{i} f\right)(g)=f\left(g \odot g_{i}\right)
$$

which is nothing but the difference operator on $\mathcal{A}$, and satisfies

$$
\partial_{i} \partial_{j}=\sum_{k} C_{i j}^{k} \partial_{k}, \quad C_{i j}^{k}=\delta_{i}^{k}+\delta_{j}^{k}-\delta_{i \cdot j}^{k}
$$

where $i, j, \cdots,(i \cdot j)$ denote $g_{i}, g_{j}, \cdots,\left(g_{i} \cdot g_{j}\right)$ respectively. The basis $\chi^{i}$ of $\Omega^{1}$ are just the dual of $\partial_{i}$,

$$
\chi^{i}\left(\partial_{j}\right)=\delta^{i}{ }_{j}
$$

Then the first order differential calculus $\left(\Omega^{1}, d\right)$ is given by

$$
d f=\sum_{i=1}^{N_{G}-1} \partial_{i} f \chi^{i}
$$

For the differential algebra $\Omega^{*}$ over $\mathcal{A}$ and exterior derivative, 


$$
d: \Omega^{n} \rightarrow \Omega^{n+1}
$$

satisfies the nilpotency and the graded Leibniz rule

(i) $\quad d^{2}=0$

$$
\text { (ii) } \quad d(f g)=d f \cdot g+(-1)^{\operatorname{deg} f} f \cdot d g, \quad \forall f, g \in \Omega^{*},
$$

could be obtained provided that $\chi^{i}$ satisfy the following two conditions,

$$
\begin{aligned}
& \chi^{i} f=\left(R_{i} f\right) \chi^{i}, \quad f \in \mathcal{A}, \\
& d \chi^{i}=-\sum_{j, k} C_{j k}^{i} \chi^{j} \otimes \chi^{k}, \quad g \in G^{\prime} .
\end{aligned}
$$

The involution operator $*$ on the differential algebra $\Omega^{*}$ is well defined if it agrees with the complex conjugation on $\mathcal{A}$, takes the assumption that $\left(\chi^{g}\right)^{*}=-\chi^{g^{-1}}$, and (graded) commutes with d, i.e. $d\left(\omega^{*}\right)=(-1)^{\operatorname{deg} \omega}(d \omega)^{*}$. The integral, which remains invariant under the group action, is introduced as a complex valued linear functional on $\mathcal{A}$ as,

$$
\int_{G} f=\frac{1}{N_{G}} \sum_{g \in G} f(g) .
$$

Let us consider the case that there are Lie group transformations among the elements of the function space and those transformations also depend on the elements of the discrete group. Then the derivatives introduced above are no longer covariant. In order to get meaningful differential calculus in this case, the connection one form is needed to define the covariant exterior differential:

$$
D=d_{G}+\phi
$$

where the connection one form $\phi$ may be written as

$$
\phi=\phi_{g} \chi^{g}
$$

from which we get the generalized curvature two form

$$
F=A+A \otimes A=\sum_{g, h} F_{g h} \chi^{g} \otimes \chi^{h}
$$

where

$$
F_{g h}=\partial_{g} \phi_{h}+\phi_{g} R_{g} \phi_{h}-C_{g} h^{k} \phi_{k} .
$$

This formula is simpler in terms of $\Phi=1-\phi$

$$
F_{g} h=P g i_{g} R_{g}\left(\Phi_{h}\right)-\Phi_{h \otimes g} .
$$


After introducing the the metric, we can get the Lagrangian for the theory.

For the $Z_{2}$ case, we can define the metric as

$$
<\chi, \chi>=\eta, \quad<\chi \otimes \chi, \chi \otimes \chi>=\eta^{2}
$$

then

$$
\mathcal{L}=-<\bar{F}, F>=-\eta^{2}\left(\Phi \Phi^{\dagger}-1\right)^{2}
$$

This is of Higgs potential type up to some coupling constants. To get the entire Lagrangian of the Higgs, we need to consider the space-time part. For detail it is referred to $[1,5]$.

\section{References}

1. H.G. Ding, H.Y. Guo, J.M. Li and K. Wu, Comm. Theor. Phys. 21 (1994) 8594; Higgs as gauge fields on discrete groups and standard models for electroweak and electroweak-strong interactions; To appear in Z.Phsik. C.

2. H.G. Ding, H.Y. Guo, J.M. Li and K. Wu, J. Phys. A 27 (1994) L75-L79; ibid. L231-L236.

3. H.Y. Guo and J.M. Li, An $S U(2)$ generakized gauge field model with Higgs mechanism; Preprint ASITP-94-40; July, 1994.

4. A. Connes, Non-Commutative Geometry English translation of Geometrie Non-Commutative, IHES Paris, Interedition.

5. A. Sitarz, Non-commutative Geometry and Gauge Theory on Discrete Groups, preprint TPJU-7/1992.

6. A. Connes, in: The Interface of Mathematics and Particle Physics, eds. D. Quillen, G. Segal and S. Tsou (Oxford U. P, Oxford 1990).

7. A. Connes and J. Lott, Nucl. Phys. (Proc. Suppl.) B18, 44 (1990).

8. A. Connes and Lott, Proceedings of 1991 Cargese Summer Conference.

9. D. Kastler, Marseille, CPT preprint CPT-91/P.2610, CPT-91/P.2611. 
10. A. H. Chamseddine, G Felder and J. Fröhlich, Phys. Lett. 296B (1993) 109, Zurich preprint ZU-TH-30/92 and Zurich preprint ETH-TH/92/44. A. H. Chamseddine and J. Fröhlich, $S O(10)$ Unification in Noncommutative Geometry, ZU-TH-10/1993.

11. R. Coquereaux, G. Esposito-Farése and G Vaillant, Nucl Phys B353 689 (1991).

12. E. Álvarez, J.M. Gracia-Bondia and C.P. Martin, Phys. Lett. B306, 53 (1993).

13. S.T. Hu, Homotopy Theory, Academic Press, New York, 1959. 